\title{
Research Article \\ Effect of Mild and Severe Unilateral Knee Joint Pain on Gait in Elderly Females
}

\author{
Hiroki Sugiura ${ }^{1}$ and Shinichi Demura ${ }^{2}$ \\ ${ }^{1}$ Department of Industrial Business and Engineering, Fukui University of Technology, 3-6-1 Gakuen, Fukui, Fukui 910-8505, Japan \\ ${ }^{2}$ Graduate School of Natural Science and Technology, Kanazawa University, Kakuma, Kanazawa, Ishikawa 920-1192, Japan \\ Correspondence should be addressed to Hiroki Sugiura; sugiura@fukui-ut.ac.jp
}

Received 19 June 2014; Revised 4 September 2014; Accepted 23 November 2014; Published 8 December 2014

Academic Editor: Tania Giovannetti

Copyright (c) $2014 \mathrm{H}$. Sugiura and S. Demura. This is an open access article distributed under the Creative Commons Attribution License, which permits unrestricted use, distribution, and reproduction in any medium, provided the original work is properly cited.

Gait change in the elderly may be a strategy to maintain postural stability while walking. However, gait laterality is accompanied by back pain or an increased risk of a fall. This study aimed to examine group-related differences and gait laterality in elderly females with mild or severe unilateral knee pain. Seventy-five elderly females (66-87 years old) were included, which comprised the following groups: 47 with mild unilateral knee pain and 28 with severe unilateral knee pain. They completed a $12 \mathrm{~m}$ walk test with maximum effort. Stance time, swing time, and step length were selected as evaluation parameters. A two-way ANOVA (group $\times$ leg) was used for analysis. No significant differences were found in interaction or in either main factor of the group and leg. In conclusion, elderly females do not show group-related differences or gait laterality regardless of the degree (mild or severe) of unilateral knee pain.

\section{Introduction}

Walking is the most basic movement in daily life [1]. In old age, walking can be greatly affected by a marked decline in physical functions such as leg strength, balance, and leg joints. As a result, the frequency of activities of daily living (ADL) becomes markedly limited [2], quality of life (QOL) decreases [3], and the risk of fall increases [4]. It is therefore important to prevent a decrease of the aforementioned physical parameters in the elderly.

Arthritis-associated knee joint pain affects locomotion in the elderly, particularly the female elderly. They have difficulty in walking or standing up $[5,6]$. There are considerable individual differences in the degree of these conditions even among the elderly with knee joint pain. Tennant et al. [7] reported that approximately $8 \%$ of elderly individuals have severe knee pain requiring regular visits to a hospital or hospitalization. On the other hand, the elderly with mild knee pains who do not require regular visits are considered to be an auxiliary group of the severe knee pain group $[8,9]$. This subset accounts for approximately $20 \%$ of the elderly [10-12]. Astephen et al. [13] and Sugiura and Demura [14] examined the effect of the degree of knee pain on gait and reported that the elderly with severe knee pain have inferior walking ability compared to those with mild knee pain.

Walking is a symmetric and rhythmical exercise. The elderly with unilateral knee pain may have difficulty in exerting strength in the affected leg $[5,15]$, leading to gait laterality. Gait laterality in the elderly is a strategy to maintain stability during walking. However, it is accompanied by back pain [9] or an increased risk of fall [16]. Gait laterality in people with chronic unilateral cerebral infarction [17] or people with severe unilateral leg pain [18] has been reported, but few reports exist regarding the elderly who can independently perform ADL. It is hypothesized that the mild and severe unilateral knee pain groups have gait laterality, and gait is different from former and latter groups. Gait laterality may cause a decrease in ADL and/or QOL in the elderly, even if they could independently perform ADL. In addition, laterality is defined as the difference between "healthy and pain legs" or "right and left legs" in this study.

Peat et al. [8] reported that the prevalence of mild and severe knee pain is high in the female elderly. It is inferred that the number of elderly females with mild or severe knee 
TABLE 1: Basic statistics of age, height, and body weight in the G1 and G2 groups and the test results.

\begin{tabular}{|c|c|c|c|c|c|c|c|c|c|c|}
\hline & \multicolumn{4}{|c|}{ G1 } & \multicolumn{4}{|c|}{ G2 } & \multirow{2}{*}{$t$} & \multirow{2}{*}{$P$} \\
\hline & $\mathrm{M}$ & $\mathrm{SD}$ & MAX & MIN & $\mathrm{M}$ & $\mathrm{SD}$ & MAX & MIN & & \\
\hline Age (yr) & 76.0 & 4.8 & 85 & 66 & 77.8 & 4.0 & 87 & 67 & 1.63 & 0.11 \\
\hline Height (cm) & 148.8 & 4.6 & 159.0 & 140.0 & 146.6 & 5.5 & 156.0 & 134.4 & 1.72 & 0.09 \\
\hline Body weight $(\mathrm{kg})$ & 49.5 & 7.9 & 64.9 & 32.5 & 53.1 & 6.2 & 63.9 & 39.0 & 1.84 & 0.07 \\
\hline
\end{tabular}

Note. M: mean value, SD: standard deviation, MAX: maximal value, MIN: minimum value, and $t: t$-value.

G1: mild unilateral knee pain group $(n=47)$; G2: severe unilateral knee pain group $(n=28)$.

pain will increase. This study aimed to examine group-related differences and gait laterality among elderly females with mild or with severe unilateral knee pain.

\section{Method}

2.1. Subjects. In this study, elderly individuals were asked "Do you have an articular disorder (ankle, knee, and hip joints)?" (options: yes, no) and "Do you have pain or disorders in either knee joint?" (options: right, left, both, and none); those that responded "yes" to the former question and either "right" or "left" to the latter were defined as patients with knee pain. Knee pain was determined as mild or severe as per the Japanese edition of the knee function scale [19] on the basis of the Western Ontario and McMaster Universities Osteoarthritis Index [20]. According to this assessment, individuals with over 210 points and those with less than 210 points were judged to have severe knee pain and mild knee pain, respectively [21].

Subjects comprised the following 2 groups: 47 women with mild unilateral knee pain (age: $75.8 \pm 4.9$ years, height: $148.8 \pm 4.6 \mathrm{~cm}$, and body weight: $49.6 \pm 7.9 \mathrm{~kg}$ ) and 28 persons with severe unilateral knee pain (age: $77.8 \pm 4.0$ years, height: $146.6 \pm 5.5 \mathrm{~cm}$, and body weight: $53.1 \pm 6.2 \mathrm{~kg}$ ). There were not the elderly with leg pain except for knee joint. The subjects attended health classes or social educational activities hosted by municipal governments and engaged in social activities at least once per week or alternate weeks. These parameters were used to show that they could independently perform ADL. All subjects did not do the taking medicine for pain or CNS. The purpose and procedures of this study were explained in detail to all participants, and informed consent was obtained. The study protocol was approved by the Ethics Committee on Human Experimentation of the Faculty of Human Science, Kanazawa University (Ref. No. 2012-08).

2.2. Gait. Gait properties were measured using a gait analysis system (WalkWay MG-1000; Anima, Tokyo, Japan). MG-1000 with plate sensors determines the time, dimensions, and the distance of the feet when they touch the sheet surface. It can also measure grounding/nongrounding on the bearing surface as an on/off signal. Data were recorded into a personal computer at $100 \mathrm{~Hz}$. The posture and movement during the measurements were explained to the subjects before taking them. The walkway was $12 \mathrm{~m}$ in length. The subjects walked straight for $12 \mathrm{~m}$ as fast as possible. Data from the middle $5 \mathrm{~m}$ portion, excluding the first $3 \mathrm{~m}$ and the final $4 \mathrm{~m}$, were used for analysis. The following gait parameters measured stance time, swing time, and step length for an examination by Shigeshima et al. [22]. Stance time equals the duration that one leg supports the body, that is, the phase during which one foot contacts the floor. Swing time equals the duration of time that one foot swings, that is, when it is not on the floor. Swing time is the same as single support time. Step length is the distance between anterior and posterior patterns.

2.3. Statistical Analysis. The mean differences between age, height, and body weight were examined by unpaired $t$-test, and those among groups and laterality were examined by a two-way ANOVA (group $\times$ leg) for gait parameters. The significance level in this study was set at $P<0.05$.

\section{Results}

Table 1 shows the basic statistics of age, height, and body weight in both groups, mild (G1) and severe unilateral knee pain (G2), and the results of the statistical analyses of their means. No significant difference was found for any parameter. Table 2 shows the basic statistics in the G1 and G2 groups for gait parameters and the results of the statistical analyses of their means. No significant difference was found for any parameter.

\section{Discussion}

Approximately $50 \%$ of elderly people with orthopedic abnormalities do not feel knee pain $[8,23]$. Peat et al. [8] reported that it is necessary to focus on pain in the knee joints because many elderly people have it and the type and cause vary. We therefore focused on the presence or absence of subjective knee joint pain in this study, the degree of which was determined based on a subjective judgment of each subject.

It is thought that the gait of elderly people with severe unilateral knee pain is different from that of elderly people with mild unilateral knee pain because the former strongly avoids the knee pain and has a decrease in total physical active mass. Astephen et al. [13] reported that both these groups have different stance times and step lengths. However, the present results did not show group-related differences in gait parameters. Murray et al. [24] reported a strong relationship between knee extension strength and gait. Sugiura and Demura [14] found an insignificant difference in knee extension strength between the elderly with mild knee pain and those with severe knee pain that also had high independence in ADL. In spite of having severe knee pain, 
TABLE 2: Basic statistics of each gait parameter in the G1 and G2 groups and the test results.

\begin{tabular}{|c|c|c|c|c|c|c|c|c|c|c|c|c|}
\hline & & \multicolumn{4}{|c|}{ Pain leg } & \multicolumn{4}{|c|}{ Healthy leg } & & \multicolumn{2}{|c|}{ ANOVA } \\
\hline & & $\mathrm{M}$ & $\mathrm{SD}$ & MAX & MIN & $\mathrm{M}$ & $\mathrm{SD}$ & MAX & MIN & & $F$ & $P$ \\
\hline \multirow{3}{*}{ Stance time (s) } & G1 & 0.46 & 0.06 & 0.62 & 0.34 & 0.45 & 0.05 & 0.57 & 0.35 & $\mathrm{~F} 1$ & 0.31 & 0.58 \\
\hline & G2 & 0.47 & 0.06 & 0.62 & 0.36 & 0.46 & 0.07 & 0.64 & 0.36 & $\mathrm{~F} 2$ & 3.71 & 0.06 \\
\hline & & & & & & & & & & IN & 1.37 & 0.25 \\
\hline \multirow{3}{*}{ Swing time (s) } & G1 & 0.34 & 0.03 & 0.39 & 0.28 & 0.34 & 0.03 & 0.42 & 0.27 & F1 & 0.15 & 0.70 \\
\hline & G2 & 0.34 & 0.04 & 0.43 & 0.27 & 0.34 & 0.04 & 0.41 & 0.27 & $\mathrm{~F} 2$ & 0.19 & 0.66 \\
\hline & & & & & & & & & & IN & 0.001 & 0.97 \\
\hline \multirow{3}{*}{ Step length $(\mathrm{cm})$} & G1 & 64.40 & 7.31 & 87.00 & 53.00 & 63.87 & 7.13 & 76.50 & 47.88 & $\mathrm{~F} 1$ & 2.21 & 0.14 \\
\hline & G2 & 62.71 & 7.86 & 82.50 & 50.63 & 61.17 & 7.65 & 73.67 & 45.00 & $\mathrm{~F} 2$ & 2.32 & 0.13 \\
\hline & & & & & & & & & & IN & 0.67 & 0.42 \\
\hline
\end{tabular}

Note. M: mean value, SD: standard deviation, MAX: maximal value, and MIN: minimum value.

F1: group (G1, G2), F2: leg (pain, healthy), IN: interaction, and F: F-value.

G1: the mild unilateral knee pain group $(n=47)$; G2: the severe unilateral knee pain group $(n=28)$.

subjects in this study could independently perform ADL. Thus, difference in knee extension strength between the two groups may not have been significant. Furthermore, our study only evaluated elderly people with unilateral knee pain, which may have led to findings different from these reports that included elderly people with bilateral knee pain. It is also possible that the very short evaluated walking distance $(12 \mathrm{~m})$ allowed them to walk while enduring knee pain. A different result may have been obtained if walkway was extended or normal walking speed was used. Group-related differences in gait were not found in either leg regardless of the degree of unilateral knee pain (mild or severe) in this group of elderly people who could walk independently.

The results of the present study did not find gait laterality in the group with mild unilateral knee pain. Shigeshima et al. [22] reported that laterality was not found in stance time, swing time, or step length in healthy people. Sugiura and Demura [15] reported that laterality was not found in stance time, swing time, step length, and swing speed in the elderly without knee pain, with mild unilateral or bilateral knee pain. These studies suggest that, similar to young people without leg disorders or elderly people without knee pain, the elderly with unilateral mild knee pain have little laterality in gait. Maruyama [25] reported that knee joint pain occurs mainly in scenarios such as the initiation of movement, standing up and sitting down, and stair ascent and decent, but the pain is somewhat reduced during level ground gait. It is possible that the elderly with mild unilateral pain could walk while enduring knee pain.

Sugiura and Demura [15] and Sugiura et al. [5] reported that laterality was found in the knee extension strength of elderly people with both mild and severe unilateral knee pain. Because a decrease in knee extension strength affects walking, it was assumed that laterality would be found in the gait of elderly people with mild and severe unilateral knee pain. However, none of the parameters evaluated in the present studied were consistent with this assumption. The elderly people in this study with severe unilateral knee pain had a high level of independence in ADL. It is thus believed that they could walk while enduring knee pain because of the very short distance used in this study. Demura et al. [26] reported that the gait change in elderly people is a type of strategy to maintain stability during walking. However, it was found that the elderly with severe unilateral knee pain were able to endure knee pain and to maintain stability during walking without gait laterality.

Kirsten [27] and Maruyama [25] reported that when knee joint pain increases, symptoms such as consistent pain or flexural contracture appear and the gait changes. Titianova and Tarkka [17] reported that people with chronic unilateral cerebral infarction have gait laterality. Sadeghi et al. [18] reported that people with severe unilateral leg pain have laterality in step length. These studies highlight that the gait of both legs might be different if the elderly were not able to independently accomplish ADL and have a serious disorder only in one knee. In addition, when experimental conditions (e.g., distance of the walkway, waking speed) differ, gait laterality may occur even if the elderly could independently perform ADL.

\section{Conclusion}

Group-related differences in gait were not found in either leg of female elderly people who could perform ADL independently, regardless of the degree of unilateral knee pain (mild or severe). Gait laterality was not found in either group. Laterality may not result in a change in gait in the elderly when walking a short distance, even if they have the unilateral knee pain.

\section{Conflict of Interests}

The authors declare that there is no conflict of interests regarding the publication of this paper.

\section{References}

[1] D. J. Pratt, "Some aspects of modern orthotics," Physiological Measurement, vol. 15, no. 1, pp. 1-27, 1994. 
[2] J. Stessman, R. Hammerman-Rozenberg, A. Cohen, E. EinMor, and J. M. Jacobs, "Physical activity, function, and longevity among the very old," Archives of Internal Medicine, vol. 169, no. 16, pp. 1476-1483, 2009.

[3] M. Suzuki, N. Ohyama, K. Yamada, and M. Kanamori, "The relationship between fear of falling, activities of daily living and quality of life among elderly individuals," Nursing \& Health Sciences, vol. 4, no. 4, pp. 155-161, 2002.

[4] M. E. Tinetti, "Preventing falls in elderly persons," The New England Journal of Medicine, vol. 348, no. 1, pp. 42-49, 2003.

[5] H. Sugiura, S. Demura, and K. Takahashi, "Evaluating the effects of pain and disorders of the knee joint on knee extension strength and daily life activities in the female elderly," Pain Studies and Treatment, vol. 1, no. 3, pp. 17-23, 2013.

[6] H. Sugiura and S. Demura, "Effects of mild and severe knee joint pain on various activities of daily living in the female elderly," Pain Research and Treatment, vol. 2013, Article ID 989508, 10 pages, 2013.

[7] A. Tennant, J. Fear, A. Pickering, M. Hillman, A. Cutts, and M. A. Chamberlain, "Prevalence of knee problems in the population aged 55 years and over: identifying the need for knee arthroplasty," British Medical Journal, vol. 310, no. 6990, pp. 1291-1293, 1995.

[8] G. Peat, R. McCarney, and P. Croft, "Knee pain and osteoarthritis in older adults: a review of community burden and current use of primary health care," Annals of the Rheumatic Diseases, vol. 60, no. 2, pp. 91-97, 2001.

[9] Z. Bejek, R. Paróczai, Á. Illyés, and R. M. Kiss, “The influence of walking speed on gait parameters in healthy people and in patients with osteoarthritis," Knee Surgery, Sports Traumatology, Arthroscopy, vol. 14, no. 7, pp. 612-622, 2006.

[10] T. E. McAlindon, S. Snow, C. Cooper, and P. A. Dieppe, "Radiographic patterns of osteoarthritis of the knee joint in the community: the importance of the patellofemoral joint," Annals of the Rheumatic Diseases, vol. 51, no. 7, pp. 844-849, 1992.

[11] S. C. O'Reilly, K. R. Muir, and M. Doherty, "Screening for pain in knee osteoarthritis: which question?" Annals of the Rheumatic Diseases, vol. 55, no. 12, pp. 931-933, 1996.

[12] M. Urwin, D. Symmons, T. Allison et al., "Estimating the burden of musculoskeletal disorders in the community: the comparative prevalence of symptoms at different anatomical sites, and the relation to social deprivation," Annals of the Rheumatic Diseases, vol. 57, no. 11, pp. 649-655, 1998.

[13] J. L. Astephen, K. J. Deluzio, G. E. Caldwell, and M. J. Dunbar, "Biomechanical changes at the hip, knee, and ankle joints during gait are associated with knee osteoarthritis severity," Journal of Orthopaedic Research, vol. 26, no. 3, pp. 332-341, 2008.

[14] H. Sugiura and S. Demura, "The effects of knee joint pain and disorders on knee extension strength and walking ability in the female elderly," Advances in Physical Education, vol. 2, no. 4, pp. 139-143, 2012.

[15] H. Sugiura and S. Demura, "Effect of subjective knee-joint pain on the laterality of knee extension strength and gait in elderly women," Archives of Gerontology and Geriatrics, vol. 54, no. 2, pp. 366-369, 2012.

[16] H. Kim, T. Suzuki, H. Yoshida et al., "Are gait parameters related to knee pain, urinary incontinence and a history of falls in community-dwelling elderly women?" Japanese Journal of Geriatrics, vol. 50, no. 4, pp. 528-535, 2013.

[17] E. B. Titianova and I. M. Tarkka, "Asymmetry in walking performance and postural sway in patients with chronic unilateral cerebral infarction," Journal of Rehabilitation Research and Development, vol. 32, no. 3, pp. 236-244, 1995.

[18] H. Sadeghi, P. Allard, F. Prince, and H. Labelle, "Symmetry and limb dominance in able-bodied gait: a review," Gait \& Posture, vol. 12, no. 1, pp. 34-45, 2000.

[19] H. Hashimoto, T. Hanyu, C. B. Sledge, and E. A. Lingard, "Validation of a Japanese patient-derived outcome scale for assessing total knee arthroplasty: comparison with Western Ontario and McMaster Universities osteoarthritis index (WOMAC)," Journal of Orthopaedic Science, vol. 8, no. 3, pp. 288-293, 2003.

[20] N. Bellamy, W. W. Buchanan, C. H. Goldsmith, J. Campbell, and L. W. Stitt, "Validation study of WOMAC: a health status instrument for measuring clinically important patient relevant outcomes to antirheumatic drug therapy in patients with osteoarthritis of the hip or knee," Journal of Rheumatology, vol. 15, no. 12, pp. 1833-1840, 1988.

[21] Y. Oida and N. Nakamura, Exercise Learning Support Manual for the Knee Joint Ache Prevention and Reduction, Sunlife Plan, Tokyo, Japan, 2008.

[22] K. Shigeshima, T. Fujiwara, Y. Ogoma, M. Ohkura, and H. Nakaya, "Symmetry of step length and temporal variability in gait of people without impairment," Journal of Japan Academy of Health Sciences, vol. 12, no. 1, pp. 25-30, 2009.

[23] P. A. Dieppe and L. S. Lohmander, "Pathogenesis and management of pain in osteoarthritis," The Lancet, vol. 365, no. 9463, pp. 965-973, 2005.

[24] M. P. Murray, E. H. Duthie Jr., S. R. Gambert, S. B. Sepic, and L. A. Mollinger, "Age-related differences in knee muscle strength in normal women," Journals of Gerontology, vol. 40, no. 3, pp. 275-280, 1985.

[25] H. Maruyama, The Gait, The Society of Physical Therapy Science, Tokyo, Japan, 2003.

[26] S. Demura, S. Yamaji, and S. Sato, "Gait and fall characteristics of the elderly," Journal of Joint Surgery, vol. 30, no. 2, pp. 100-107, 2011.

[27] G. Kirsten, Gait Analysis by Observation, Igaku-Shoin, Tokyo, Japan, 2009. 


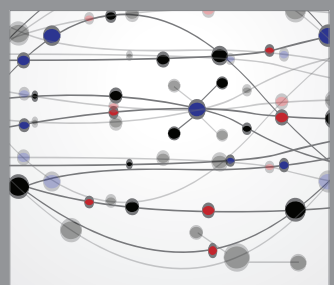

The Scientific World Journal
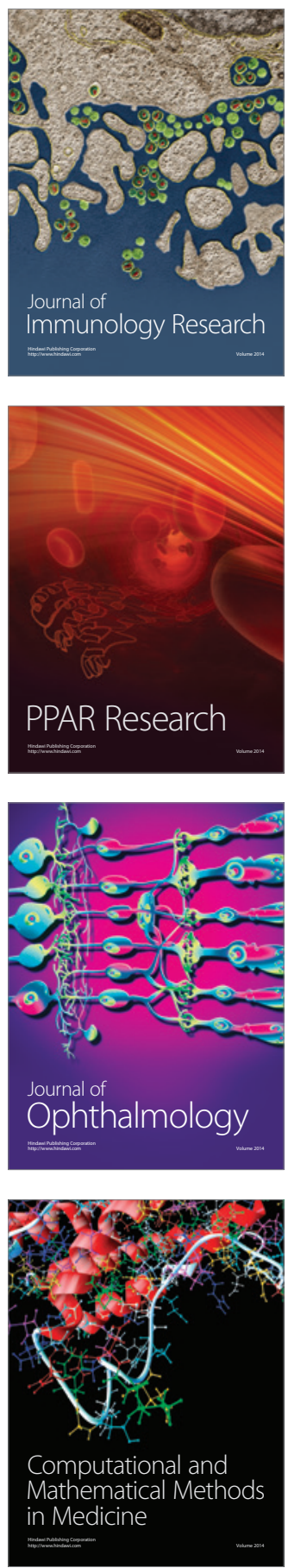

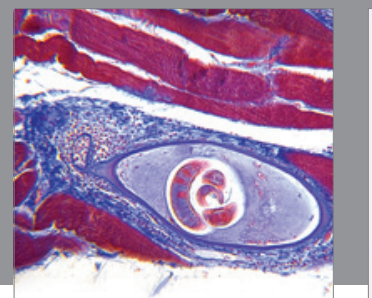

Gastroenterology

Research and Practice
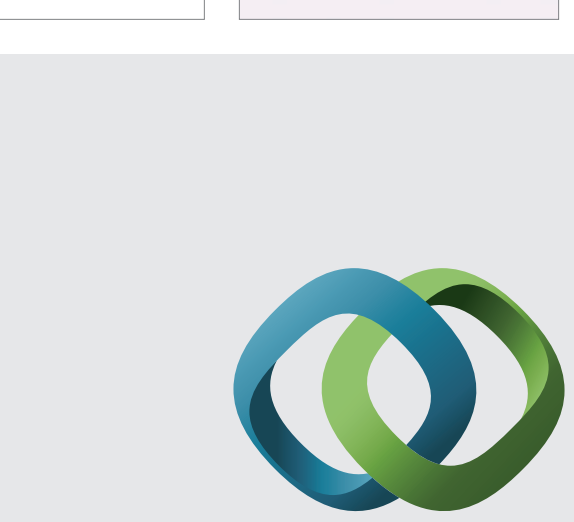

\section{Hindawi}

Submit your manuscripts at

http://www.hindawi.com
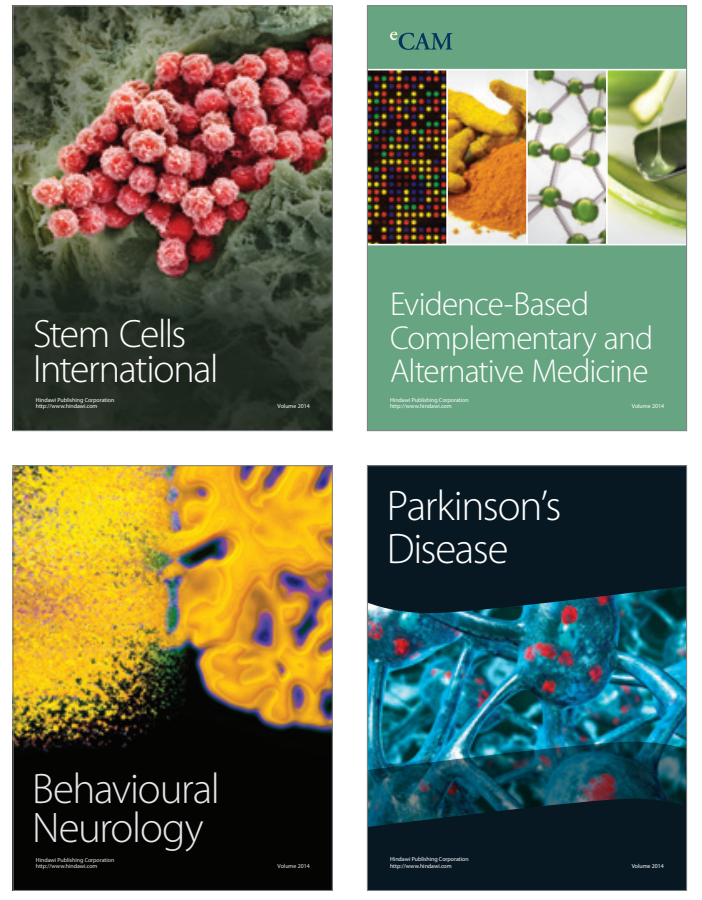
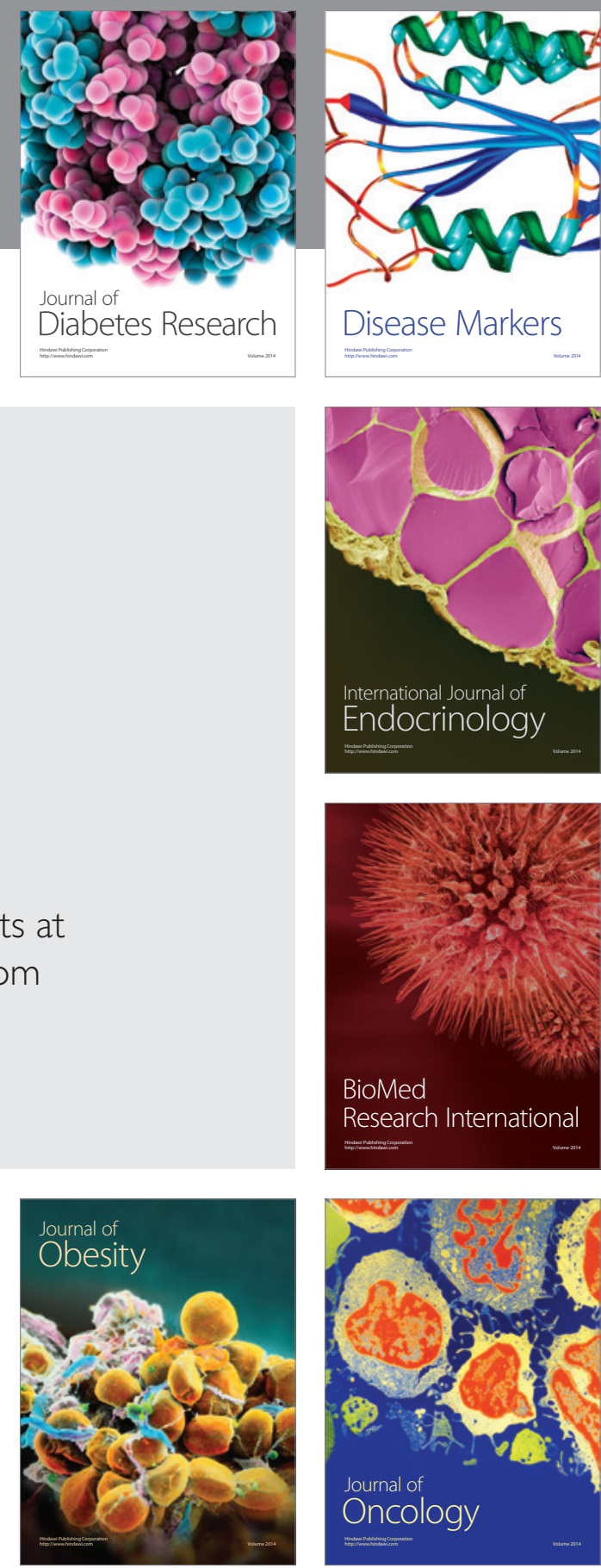

Disease Markers
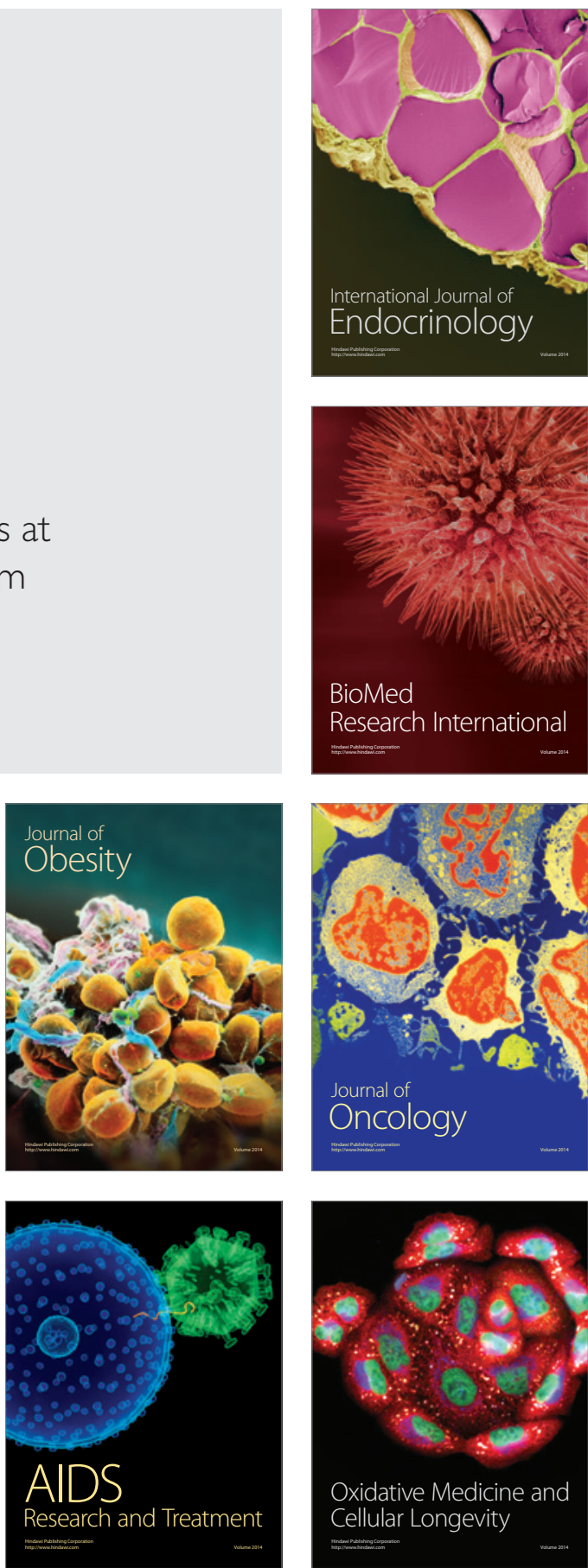